\title{
Research on recognition method of vehicle lane-change behavior based on video image
}

\author{
Rongbao Chen ${ }^{1 \mathrm{a}}$,Xiaoer $\mathrm{Ye}^{1 \mathrm{~b}}$, Feng-yan Zhang ${ }^{23}$,Dan Zhao ${ }^{1 \mathrm{c}}$ \\ ${ }^{1}$ School of Electrical Engineering and Automation, Hefei University of Technology, Hefei \\ 230001, China \\ ${ }^{2}$ School of Transportation, Southeast University, Nanjing 210096,China \\ ${ }^{3}$ Jiangsu Transportation Institute, Nanjing 210017, China \\ acrbwish@126.com, byexiaoer@yeah.net, ${ }^{\mathrm{c}}$ zhd1553679557@163.com
}

Keywords: video image processing technology, HOUGH , HOG\&SVM, lane change.

\begin{abstract}
The research statistics show that about 60\%-70\% traffic accidents are caused by vehicle collisions. And lane change as the most common behavior when driving a vehicle is also the main reason for vehicle collisions. This paper based on the theories of video image processing technology, adopts the improved method of HOUGH to extract and fit the lane line. Through multi-threshold setting we determine the underneath shadow and further position the region of interest of the vehicle (ROI). In the case of offline, take features of large number of positive and negative HOG samples, and use the SVM to do the classified training. After extracting the HOG features, combine them with SVM classifier to achieve recognition of the vehicle. Then position the center of the vehicle, and use the binocular ranging method to get the distance between the vehicle and the lane lines. Finally, calculate the dispersion of the distance and set appropriate thresholds and determine whether the vehicle have the lane change behavior.
\end{abstract}

\section{Introduction}

With the increase accidents in traffic, there is a growing emphasis on the traffic safety. In order to reduce traffic accidents, many countries have begun to develop intelligent transportation. (Intelligent Transport System, ITS)[1]. The research statistics show that about $60 \%-70 \%$ traffic accidents are caused by vehicle collisions. And lane change as the most common behavior when driving a vehicle is also the main reason for vehicle collisions. German Daimler-Benz research reports that if the driver can get more 0.5 seconds of warning time that $60 \%$ of rear-end accidents can be avoided, if more one second of warning time $90 \%$ of rear-end accidents can be avoided[2]. So the identification of the vehicle lane change behavior is important for auxiliary driving.

The research of domestic and foreign scholars on the vehicle change lanes, are mostly limited to the lane change model based on traffic flow macroscopic and microscopic simulation or lane change warning and simplify the automatic lane change control. About the practical applications, mostly are based on radar and a variety of sensors, vehicle monitoring. Radar sensors and related equipment are not only expensive but also inconvenient to install. Therefore it is difficult to be got substantial promotion. The technology based on video image processing in the field of intelligent transportation has been developed greatly. The video equipment is not only easy to install, and with the rapid development of computer processing speed, real-time image processing has also been greatly improved. The method on image processing has a great advantage on the aspect of obtaining vehicle lane change behavior.

The paper based on the image processing technology and combined with the recognition of shadow, recognizes the ROI region and uses the HOG features and SVM classifier to complete the identification of the vehicle. Finally, calculate the dispersion of the distance and set appropriate thresholds and determine whether the vehicle have the lane change behavior. 


\section{The pretreatment of image}

The remote vehicles in the image have little effect on the current vehicle when you are driving. Therefore, we could ignore the remote vehicles in the image. The upper part of the image that contains some of the remote vehicles and sky, in the process of image preprocessing can intercept the lower half of the image for processing. We can assume that the image height is h, select $0 \mathrm{~h} \sim 2$ / 3h as the pretreatment portion.

The vehicle video system captures images which will be affected by the light, camera, vehicle bumps and other reasons. That will be results various noise in the images and then will have some influence on the subsequent analysis. So before image analysis, it is need to do preprocessing for image. This paper pretreatment includes: an image cropped, graying, smoothing, edge detection. As shown in Figure 1.

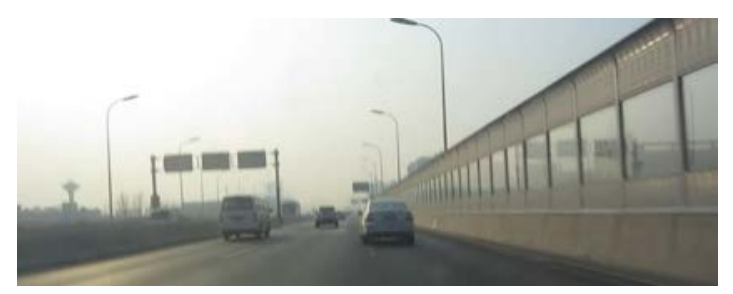

(a)original image

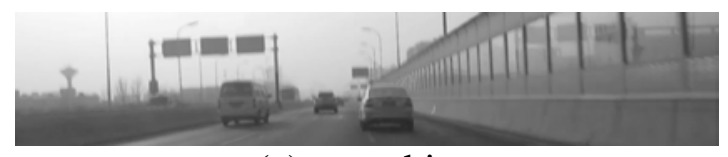

(c)smoothing

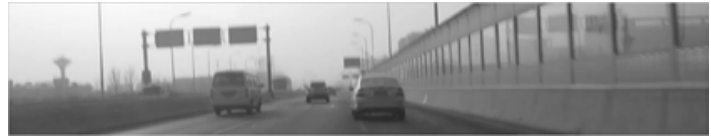

(b)image cropped,and graying

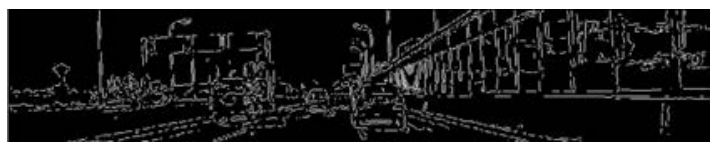

(d)edge detection

Fig1. Image preprocessing

\section{ROI of Image}

To avoid searching the entire image, we need to extract the ROT (Region of Interest) form the image which had pre-processed that could decrease computing time. Typically the gray value of shadow is smaller than the pavement. Document [3] obtains vehicle bottom shadow position by the Equation (1), and estimates vehicle initial position.

$$
G(\mathrm{i}, \mathrm{j})=\left\{\begin{array}{lc}
0 & f(i, j) \geq T H \\
255 & \text { other }
\end{array}\right.
$$

Choosing the threshold of the shadow is the key to the shadow extraction. Using a fixed threshold is difficult to ensure the shadow recognition performance under different lighting conditions. This paper uses the method based on pavement gray levels to identify shadows. We can analysis the gray value histogram of the pavement area that is similar to normal distribution .As shown in Equation (2).a. We can set threshold according to Equation (2).b after getting the pavement area mean value $u$ and $\sigma$.

$$
F(\mathrm{x}) \approx k \cdot e^{\frac{-(x-u)^{2}}{2 \sigma^{2}}} \quad \text { (a) } \quad T H=\left\{\begin{array}{cc}
u-3 \sigma & \sigma \leq 10 \\
u-3 \sigma & 10 \leq \sigma \leq 20 \\
u-3 \sigma & 20 \leq \sigma \leq 30 \\
u-3 \sigma & \sigma>30
\end{array}\right.
$$




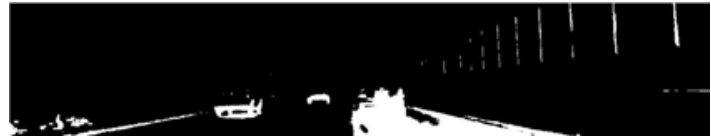

(a) fixed threshold

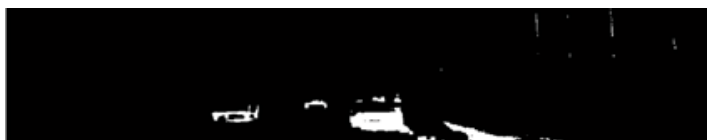

(b) multi-threshold

Fig 2 shadow extraction

As we can see from the above figure 2, Multi-Threshold shadow extraction relative to fixed threshold shadow extraction can filter more interference. Since the vehicle shadow has obvious class matrix properties and shadow pixel area has a certain range. Take the external matrix in the area. The ratio is large between Shadows and external matrix. Based on the above two points, by setting the maximum and minimum of the shadow pixel area, calculate the ratio of the suspected area with external matrix, can exclude interference area, the results as shown in Figure 3.

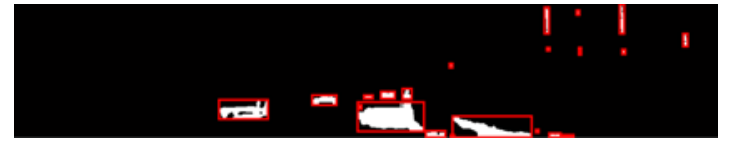

(a) all shadow external matrix

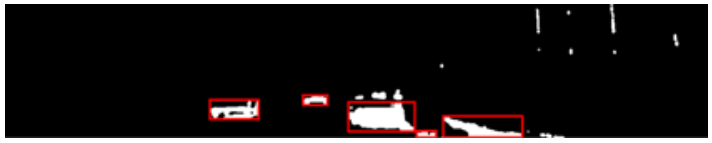

(b) eligible shadow external matrix

Fig 3 shadow external matrix

As you can see from Figure 3 (b), setting conditions can exclude more interference shadow areas. Since the position of the vehicle located above the shadow, takes the bottom edge of rectangular area as the ROI bottom edge, and takes 1.2 times the width of the rectangular area as the height of preliminary ROI, as shown in Figure 4.

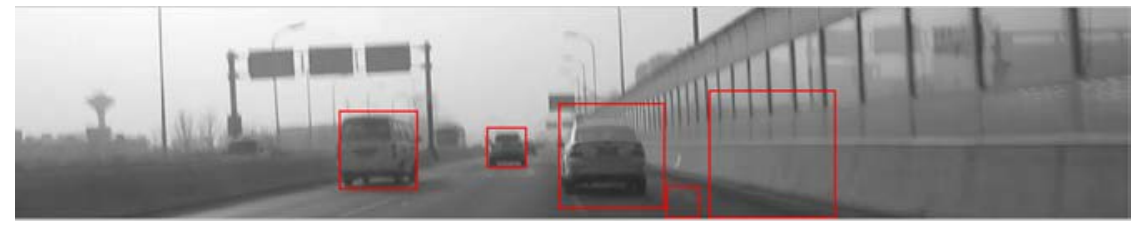

Fig 4 vehicle ROI

\section{Vehicle detection and tracking}

The traditional methods of vehicle detection and recognition depend too much on the environment and which have the low precision. The methods based on machine learning become a hot research gradually. This paper uses the support vector machine (SVM), combined with HOG characteristic complete the vehicles identification.

The core idea of HOG is that the detected partial object shape which could be described by the distribution of edge orientation or light intensity, and the HOG features affected by the light, direction and size slightly. In the vehicle images, the density distribution of the intensity or the edge can describe the appearance of the vehicles. When the gray value changes slowly, the gradient magnitude of the area is small, when the gray value changes greatly the gradient magnitude of the area is large[4] . Therefore, the HOG feature can describe the gradient edge information of target vehicle better. The gradient magnitude and gradient direction calculation formula of pixel $(\mathrm{x}, \mathrm{y})$ as follows: 


$$
\left\{\begin{array}{l}
G_{\mathrm{x}}(x, y)=H(\mathrm{x}+1, y)-H(\mathrm{x}-1, y) \\
G_{\mathrm{y}}(x, y)=H(\mathrm{x}, y+1)-H(\mathrm{x}, y-1)
\end{array}(\mathrm{a})\right.
$$

$$
\left\{\begin{array}{c}
G(x, y)=\sqrt{G_{x}(x, y)^{2}+G_{y}(x, y)^{2}} \\
\alpha(x, y)=\arctan \left[G_{y}(x, y) / G_{x}(x, y)\right]
\end{array}\right.
$$

In the Equation (3), the $H(x, y)$ is the pixel value, the and $G_{X}(x, y), G_{y}(x, y)$ is respectively the horizontal gradient and vertical gradient of the pixels which are input the images $a(x, y)$ is the gradient direction.

SVM (support vector machine) is a machine learning method based on statistical learning theory [5], by seeking an optimal hyperplane, to realize the two or more classes-classification problems. Vehicle detection problem is essentially two categories of classification, when SVM used for classification, it has a good learning accuracy and learning ability. Therefore the SVM has a good adaptability and has been widely used in vehicles, pedestrian detection classifier.

The key to use SVM is to select the kernel functions and the parameters .By comparing the results of the experiments, the paper chooses radial basis kernel function, take $\mathrm{C}=32, \sigma=128$. In the case of offline, extracting the HOG features after selecting the samples. When carrying on the real-time detection, extracting the HOG feature of the ROI which could get from last section. We can use the SVM classifier for further verification and identification of the vehicle in the ROI. By using the SVM classifier can effectively identify the vehicle regional and non-vehicular area, thereby completing the vehicle detection. The result of the verification is shown in Figure 5.

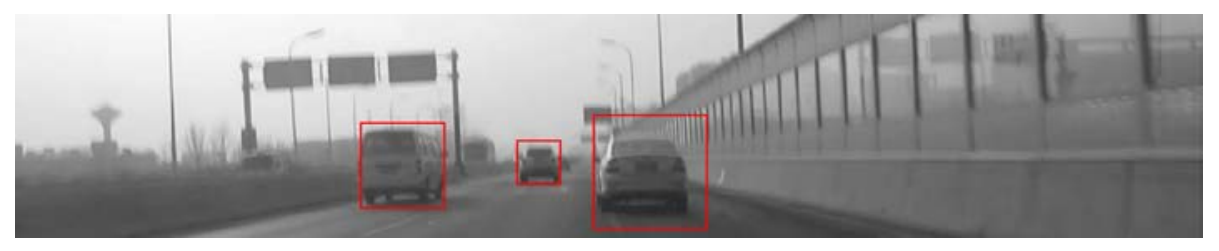

Fig 5 vehicle detection

The actual maximum distance between each adjacent frames about $1.1 \mathrm{~m}$ with the fastest speed of the vehicle $120 \mathrm{~km} / \mathrm{h}$ calculation and acquisition speed of 30 frames per second. This shows the change between the frames is not great. After recognizing by SVM, that could access the current vehicle areas of the video frame, when track the vehicle in the next frame, upper the area of the vehicle in last frame as the preselected area. This paper extracts the ROI in this area, then use the above method to further obtain the vehicle area in this frame to complete tracking the vehicle.

\section{Vehicle lane-change behavior recognition}

The distance between the vehicle and the lane is an important reference factor for recognize the vehicle lane change behavior. So it is a crucial step that obtaining the distance between the lane and the vehicle.

Hough transformation is the most common method for line detection, has good detection results for the ideal conditions, but the effect is not satisfactory in complex scenes. In this paper, an improved Hough transform [6] is used to detect lane lines. After the Hough transform, the points of the line $\left(x_{0}, y_{0}\right) 、\left(x_{1}, y_{1}\right) 、 \cdots \cdots,\left(x_{n}, y_{n}\right)$, can fit the left lane and the right lane according to the following Equation (4) : $A X+B Y+C=0, A_{1} X+B_{1} Y+C_{1}=0$.

$$
A=\frac{\sum_{i=1}^{m} y_{n i} \sum_{i=1}^{m} x_{n i}{ }^{2}-\sum_{i=1}^{m} x_{n i} \sum_{i=1}^{m} x_{n i} y_{n i}}{m \sum_{i=1}^{m} x_{n i}{ }^{2}-\left(\sum_{i=1}^{m} x_{n i}\right)^{2}}
$$

$$
B=\frac{m \sum_{i=1}^{m} x_{n i} y_{n i}-\sum_{i=1}^{m} x_{n i} \sum_{i=1}^{m} y_{n i}}{m \sum_{i=1}^{m} x_{n i}{ }^{2}-\left(\sum_{i=1}^{m} x_{n i}\right)^{2}}
$$

This paper uses the binocular CCD camera for image acquisition, and using the collected 
images for distance measurement. The schematic diagram of binocular video ranging is shown in Figure 6. In the figure, $L=B f / x_{\text {dis, }}, x_{\text {dis }}=x_{\text {right }}-x_{\text {left }}$. According to the calibration, the camera's internal parameters focal length $f$ can be obtained, and then get the distance between the object and the camera.

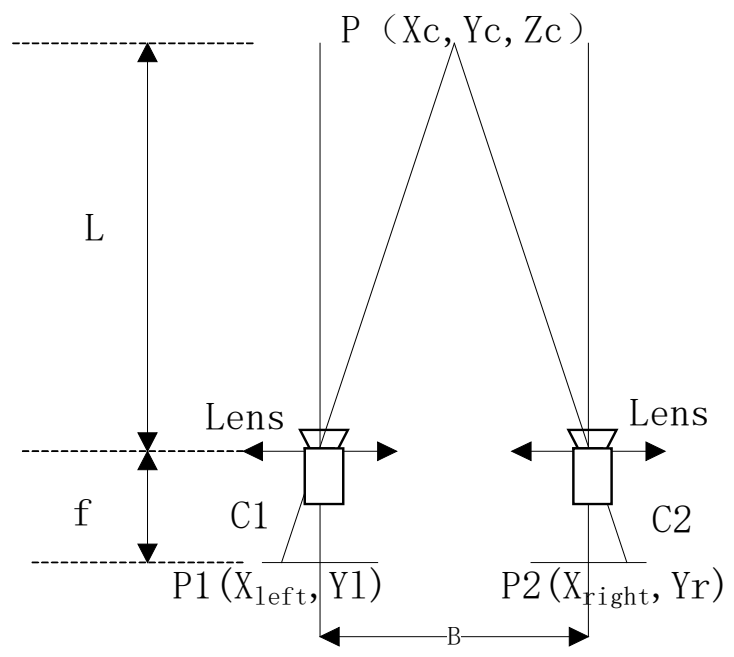

Figure 6 Binocular ranging principles

Supposing two cameras are in the same plane, so $Y_{l}=Y_{R}=Y$, from the above figure we can be drawn:

$$
\left\{\begin{array} { l } 
{ X _ { \text { left } } = f X _ { \mathrm { c } } / Z _ { c } } \\
{ X _ { \text { right } } = f ( X _ { \mathrm { c } } - B ) / Z _ { c } \quad ( \mathrm { a } ) } \\
{ \mathrm { Y } = f \mathrm { Y } _ { \mathrm { c } } / \mathrm { Z } _ { \mathrm { c } } }
\end{array} \left\{\begin{array}{l}
X_{c}=\frac{B \cdot X_{\text {left }}}{X_{\text {left }}-X_{\text {right }}} \\
Y_{c}=\frac{B \cdot Y}{X_{\text {left }}-X_{\text {right }}} \\
Z_{c}=\frac{B \cdot f}{X_{\text {left }}-X_{\text {right }}}
\end{array}\right.\right.
$$

According to the relationship between camera coordinates and world coordinates, the world coordinates can be drawn: $P_{C}=R P_{W}+T$.

When recognizing the lane-change behavior, taking the midpoint $\mathrm{M}(\mathrm{x}, \mathrm{y})$ of the bottom edge of an external matrix at the vehicle's bottom shadow. According to the relationship between world coordinates and pixel coordinates: $P_{C}=R P_{W}+T$, obtaining the world coordinates of $\mathrm{M}$ points $\left(X_{W}, Y_{W}, Z_{W}\right)$. Taking any two points $\mathrm{P} 1$ and $\mathrm{P} 2$ on the line, we can obtain the line according to the two points' world coordinates .Since the point $M$ and points $A, B$ are in the same plane. AB linear Equation can be obtained: $A X+B Y+C=0$, the Dis1 between point $\mathrm{M}$ and the left lane can be obtained, also the Dis2 of the right lane line.

$$
\operatorname{Dis} 1=\sqrt{X_{W} \cdot A+Y_{W} \cdot B+C} / A^{2}+B^{2} \text { (a) } \quad \text { Dis2 }=\sqrt{X_{W} \cdot A_{1}+Y_{W} \cdot B_{1}+C} / A_{1}^{2}+B_{1}^{2}
$$

When recognizing the behavior of vehicle Lane-change, the distance difference Dis1-Dis2 determines the vehicle change lane from the right or change lane from the left. As shown in Equation (8).

$$
\left\{\begin{array}{cc}
\text { Dis1-Dis2 }=L & (a) \\
\text { Dis2 }- \text { Dis1 }=L & (b) \\
\mid \text { Dis1-Dis2 } \mid \leq 0.8 L & (c)
\end{array}\right.
$$

$\mathrm{L}$ represents the actual width between left lane and right lane. When the Dis1-Dis2 meet with the formula (a), it can be considered that the ahead vehicle is on the left lane, in the same way, satisfy (b) may be considered that the ahead vehicle is on the right lane, when satisfying (c) can be considered that the ahead vehicle is possible to change lanes. 
The behavior that vehicle change lane is a process changed continuously. It is possible to make some misjudgment, if determining the vehicle lane-change behavior on a certain time distance from the lanes. In this paper, the ahead vehicle whether has lane-change behavior or not could be judge from the dispersion of distance between the vehicle and the lane lines. It has better reliability. Dispersion of D can be calculated according to the Equation (8).

$$
E=\frac{1}{M} \sum_{i=0}^{M} \operatorname{Dis}[i](\mathrm{a}) \quad S=\frac{1}{M} \sum_{i=0}^{M}(\operatorname{Dis}[i]-E)^{2}(\mathrm{~b}) \quad E V E N T=\left\{\begin{array}{ccc}
1 & (S>T 1) & \text { (c) } \\
0 & \text { otherwise }
\end{array}\right.
$$

By setting the threshold $\mathrm{T} 1$, when the discrete $\mathrm{S}$ is greater than $\mathrm{T} 1$, can be determined that the lane change behavior occurs at this time.

\section{Conclusion}

This paper based on video image processing technology, after obtaining images by CCD camera. Through location of underneath shadow, we determine the initial position of the vehicle in the image. Further extraction of the vehicle by using HOG features and SVM classifier; determine the position of the vehicle. Finally combined with the central position of the vehicle, Using Binocular Ranging, get the distance between the vehicle and the lane line. And through dispersion to determine whether the vehicle is changing lanes. This method has high accuracy in vehicle identifications and binocular ranging has high accuracy that has a broad application space.

\section{References}

[1] Rongbao Chen,Huafeng Xiao,Xianwei Dou,Wei Hou. Research on Recognition Methods of Bus Front Road Condition Based on Video[C].2013 Seventh International Conference on Image and Graphics(ICIG 2013):439-442.

[2] BIAN Ming-yuan. A Vehicle Safety Distance Model For Collision Avoidance System Based On Emergency Lane Change Motion[J]. Journal Of Chongqing University Of Technology,2012,26(4):1:4

[3] Xin Liu, Bin Dai, Hangen He. Real-time On-Road Vehicle Detection Combining Specific Shadow Segmentation and SVM Classification[C]. 2011 Second International Conference on Digital Manufacturing \& Automation(ICDMA 2011):885-888.

[4]ZHANG Quan-Fa,, PU Bao-Ming, LI Tian-Ran, SUN Hong-Guo.Vehicles Detection Based on Histograms of Oriented Gradients and Machine Learning [J].Computer Systems \& Applications,2013,22(7):104-107.

[5] LIU Zhenhua,HUANG Lei,LIU Changping. A Vehicle Violation Detection Algorithm Based on Video Image Processing [J]. Journal of Highway and Transportation Research and Development,2012,39(2):106-114.

[6] Chen Rongbao ,Hou Wei. Recognition Of Lanes On Complex Roads Based On Video Image[J]. Materials ,Mechatronics And Automation IV,2014,8(950):298-305. 